OPEN ACCESS

Edited by:

Huaqiang Zeng,

Northwestern Polytechnical

University, China

Reviewed by:

Debasis Manna,

Indian Institute of Technology

Guwahati, India

Zhiwei Liu,

University of the Sciences,

United States

*Correspondence:

Rebeca Garcia-Fandino rebeca.garcia.fandino@usc.es

Specialty section:

This article was submitted to Supramolecular Chemistry,

a section of the journal

Frontiers in Chemistry

Received: 01 May 2021 Accepted: 23 June 2021

Published: 27 July 2021

Citation:

Blanco-González A, Calvelo M, Garrido PF, Amorín M, Granja JR, Piñeiro Á and Garcia-Fandino R (2021)

Transmembrane Self-Assembled Cyclic Peptide Nanotubes Based on $\alpha$-Residues and Cyclic $\delta$-Amino Acids:

A Computational Study.

Front. Chem. 9:704160.

doi: 10.3389/fchem.2021.704160

\section{Transmembrane Self-Assembled Cyclic Peptide Nanotubes Based on $a$-Residues and Cyclic $\delta$-Amino Acids: A Computational Study}

\author{
Alexandre Blanco-González ${ }^{1,2}$, Martín Calvelo ${ }^{1}$, Pablo F. Garrido ${ }^{2}$, Manuel Amorín ${ }^{1}$, \\ Juan R. Granja ${ }^{1}$, Ángel Piñeiro ${ }^{2}$ and Rebeca Garcia-Fandino ${ }^{1 *}$
}

${ }^{1}$ Departamento de Química Orgánica, Center for Research in Biological Chemistry and Molecular Materials, Universidade de Santiago de Compostela, Campus Vida s/n, Santiago de Compostela, Spain, ${ }^{2}$ Departamento de Física Aplicada, Facultade de Física, Universidade de Santiago de Compostela, Santiago de Compostela, Spain

Self-assembling cyclic peptide nanotubes have been shown to function as synthetic, integral transmembrane channels. The combination of natural and nonnatural aminoacids in the sequence of cyclic peptides enables the control not only of their outer surface but also of the inner cavity behavior and properties, affecting, for instance, their permeability to different molecules including water and ions. Here, a thorough computational study on a new class of self-assembling peptide motifs, in which $\delta$-aminocycloalkanecarboxylic acids are alternated with natural $\alpha$-amino acids, is presented. The presence of synthetic $\delta$-residues creates hydrophobic regions in these $\alpha, \delta$-SCPNs, which makes them especially attractive for their potential implementation in the design of new drug or diagnostic agent carrier systems. Using molecular dynamics simulations, the behavior of water molecules, different ions $\left(\mathrm{Li}^{+}, \mathrm{Na}^{+}, \mathrm{K}^{+}, \mathrm{Cs}^{+}\right.$, and $\left.\mathrm{Ca}^{2+}\right)$, and their correspondent counter $\mathrm{Cl}^{-}$anions is extensively investigated in the nanoconfined environment. The structure and dynamics are mutually combined in a diving immersion inside these transmembrane channels to discover a fascinating submarine nanoworld where starshaped water channels guide the passage of cations and anions therethrough.

\footnotetext{
Keywords: self-assembling cyclic peptide nanotubes, transmembrane channels, molecular dynamics simulation, ion transport across lipid membrane, confined water
}

\section{INTRODUCTION}

The translocation of different species between the compartmentalized inner regions of living cells and their environment plays a core role on their viability and probability of survival. Uncontrolled exchange of matter is equivalent to a death sentence for the cell. The exquisite control of nature on this dynamic equilibrium in biological ion channels and pores provides a source of inspiration for scientists who use natural structures and functions as a reference to be mimicked and eventually improved (Tagliazucchi and Szleifer, 2015; Bogdanowicz et al., 2016).

Numerous supramolecular structures have been designed to replicate the specific functions of natural transport systems, in terms of affinity, efficiency, stability, and selectivity (Zheng et al., 2021). Among other examples, self-assembling cyclic peptide nanotubes (SCPNs) have emerged as attractive transmembrane channel mimetics due to their cylinder shape and expected biocompatibility (García-Fandiño et al., 2012b; Rodriguez-Vazquez et al., 2014; Garcia-Fandiño 


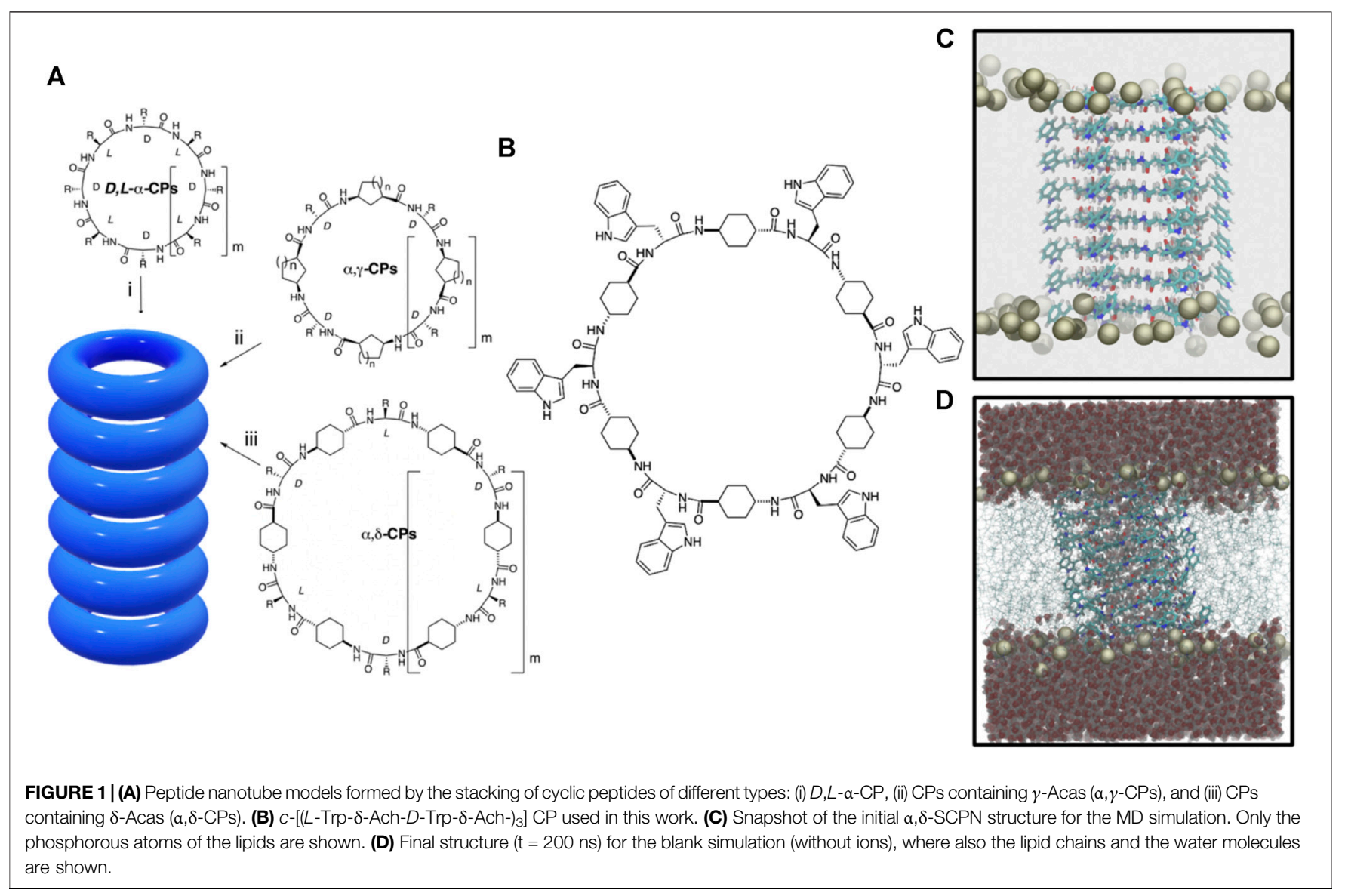

et al., 2017; Claro et al., 2018; Rodríguez-Vázquez et al., 2017). These systems were devised in 1974, but it was not until 1993 that they were first synthesized (De Santis et al., 1974; Ghadiri et al., 1993). The original SCPNs were comprised of $D$ - and $L$-a-amino acids that adopted a flat conformation in which the amide groups ( $\mathrm{NH}$ and $\mathrm{CO}$ ) lie perpendicular to the plane of the ring (Figure 1A). (De Santis et al., 1974; Bong et al., 2001) This special arrangement allows the formation of hydrogen bonds (H-bonds) between contiguous cyclic peptides (CPs) owing to the complementarity between $\mathrm{H}$-bond donor and acceptor groups on both sides of each disc structure. Thus, upon CP stacking, the interior of the assembly remains empty, with all the side chains exposed on the external surface of the cylindrical structure. One of the main advantages of these rod-shaped peptide materials is the simplicity with which their inner and outer properties can be easily modulated upon appropriate selection of the CP sequence; while the number and type of amino acids determine the internal diameter of the nanotube, the characteristics of its outer surface depend on the properties of the side chains of the amino acids. In this sense, the design of SCPNs that interact with the hydrophobic part of biomembranes has been carried out through the appropriate selection of amino acids in the basic peptide subunit (Ghadiri et al., 1994).

Most SCPNs have a hydrophilic inner cavity, designed to allow the transport of hydrophilic species (Brea et al., 2010). However, since the synthesis of the first $D, L-a-S C P N s$, SCPNs using CPs composed by different types of nonnatural amino acids have been developed, obtaining structures with more specific behavior (Montenegro et al., 2013). For example, $\beta$-SCPNs (Seebach et al., 1997; Clark et al., 1998a) are characterized by leaving all the $\mathrm{NH}$ groups in one side of the CPs and all the CO groups on the other. The resulting macrodipole leads to exotic properties such as a significant piezoelectricity (Tabata et al., 2019). Cyclic $\gamma$-amino acids (cis- $\gamma$-aminocycloalkanecarboxylic acids, $\gamma$-Acas) of appropriate chirality, both alone (Chen et al., 2020) or alternated with $\alpha$-amino acids $(\alpha, \gamma$-CPs), can also form nanotubes ( $\alpha, \gamma$-SCPNs) (Amorín et al., 2003; Garcia-Fandino et al., 2012), where the $\beta$-carbon of the cycloalkane moiety is oriented toward the lumen of the cylinder, influencing the internal properties of the nanotube and opening the possibility of inner functionalization (Figure 1A). The first steps toward the preparation and characterization of $\alpha, \gamma$-SCPNs with chemically functionalized lumens have already anticipated promising results in this area (Reiriz et al., 2009; Calvelo et al., 2015; RodríguezVázquez et al., 2017; Calvelo et al., 2019).

The behavior of water molecules and ions locked within the narrow confines of a subnanometric hydrophobic pore is very different from that of the macroscopic world. Both in vitro and in silico studies indicate that nanoconfined water inside SCPNs adopts a highly ordered structure and it shows a much slower free diffusion compared to its bulk form (Granja and Reza Ghadiri, 1994; Clark et al., 1998b; Sánchez-Quesada et al., 
2001; García-Fandiño et al., 2009; García-Fandiño et al., 2012a; Montenegro et al., 2013). This seems to be modulated by several factors such as the channel inner radius and its hydrophobicity (Montenegro et al., 2013; Rodríguez-Vázquez et al., 2016). An increase in the hydrophobic character of the nanotube can be achieved using the trans-4-aminocyclohexanecarboxylic acid $(\delta$-Ach) as a building block. The resulting nanotubes, $\alpha, \delta$-SCPNs (Lamas et al., 2018), exhibit a more hydrophobic internal cavity due to the presence of two methylene groups of each cyclohexyl moiety oriented toward the inner of the cylinder (Figure 1A). The presence of hydrophobic regions of transmembrane channels has been found to play an important role in controlling water and ion diffusion (Aryal et al., 2015; Rao et al., 2018; Yazdani et al., 2020), making $\alpha, \boldsymbol{\gamma}$ - and $\alpha, \delta$-SCPNs especially attractive for their potential implementation in the design of new drug or diagnostic agent carrier systems. While several studies have been carried out to characterize transmembrane $\boldsymbol{\alpha}, \boldsymbol{\gamma}$-SCPNs (García-Fandiño et al., 2012a; Calvelo et al., 2015; Garcia-Fandiño et al., 2016; Calvelo et al., 2019), biomimetic pores based on $\alpha, \delta$-SCPNs remain mostly uncharacterized.

Since experimental measurement about confined molecule properties in the nanoscale is difficult (Rodríguez-Vázquez et al., 2017), molecular dynamics (MD) simulations play a key role in the description of these systems (Lynch et al., 2020). Recent studies carried out in our group showed that $\alpha, \delta$-SCPNs are stable and theoretically able to translocate water and cations when inserted into a lipid bilayer (Calvelo et al., 2021). However, the behavior of the water molecules and the ions inside these structures has not been deeply examined.

This study aims to explore in detail the internal properties of transmembrane $a, \delta$-SCPN channels, evaluating and carefully investigating the structural and dynamical behavior of water, $\mathrm{LiCl}, \mathrm{KCl}, \mathrm{NaCl}, \mathrm{CsCl}$, and $\mathrm{CaCl}_{2}$ inside this type of nanotubes. The confinement effect on the structure and dynamics of water and ions in the lumen of these pores is thoroughly analyzed. The simulation results will provide new insights, useful for a better design of cyclopeptide-based structures, allowing more rational constructions for improving the transmembrane channel activity.

\section{MATERIALS AND METHODS}

The $a, \delta$-SCPN studied in this work is composed of eight CPs, long enough to traverse a previously equilibrated POPC membrane model (Calvelo et al., 2020). Each ring was made up of six $\alpha$-amino acids ( $3 L$ - and $3 D$-residues) and six $\delta$-Acas acid residues $\left\{c-\left[(L-\operatorname{Trp}-\delta-A c h-D-\operatorname{Trp}-\delta \text {-Ach- })_{3}\right]\right.$, Figure 1B $\}$. The starting geometries of the employed CPs were taken from previous works (Calvelo et al., 2021), which suggested a preferred parallel disposition of the $\beta$-sheet (Figure 1C). After nanotube insertion into the POPC bilayer, the complete system was solvated. Water molecules in the hydrophobic region of the tails and also inside the SCPN were removed, so that, in the first step of the simulation, the channel was completely dry (Figure 1D). The resulting systems were ionized using different salt solutions ( $\mathrm{LiCl}, \mathrm{NaCl}, \mathrm{KCl}, \mathrm{CsCl}$, and $\mathrm{CaCl}_{2}$ at
$0.15 \mathrm{M}$, respectively). One extra blank simulation, for control, was carried out without ions. Thus, a total of six systems were prepared.

TIP3P (Price and Brooks, 2004) was chosen as the water model, and the parameters developed by Joung et al. (Joung and Cheatham, 2008) were selected to describe the ions $\mathrm{Li}^{+}, \mathrm{Na}^{+}$, $\mathrm{K}^{+}, \mathrm{Cs}^{+}, \mathrm{Ca}^{2+}$, and $\mathrm{Cl}^{-}$, as they are known to solve some crystallization issues observed with classical parameters at relatively high concentrations. For the standard residues, the AMBER99SB-ILDN force field parameters were chosen (Lindorff-Larsen et al., 2010). For the nonstandard amino acids, RESP/6-31G(d) charges were derived, similar to those used in the development of AMBER force fields, and van der Waals parameters were obtained from the GAFF force field, all taken from a previous work (Calvelo et al., 2021). The parameters for the lipid of choice, POPC, were taken from Lipidbook (Klauda et al., 2010; Jämbeck and Lyubartsev, 2012a; Jämbeck and Lyubartsev, 2012b).

All simulations were performed with the GROMACS 2019.3 package (Abraham et al., 2015). All systems were first minimized, followed by an unrestrained production run of $200 \mathrm{~ns}$, with a time step of $2 \mathrm{fs}$. No specific restraints were applied to the peptides at any step. An NPT ensemble was employed at 1 bar using a semiisotropic Parrinello-Rahman barostat (Parrinello and Rahman, 1981) and at $300 \mathrm{~K}$ maintained with a V-rescale thermostat (Bussi et al., 2007). The LINCS algorithm was employed to remove all bond vibrations (Hess et al., 1997). Electrostatic interactions were calculated using the PME method with a cutoff of $1.0 \mathrm{~nm}$ and a grid spacing of $0.12 \mathrm{~nm}$ (Essmann et al., 1995). Van der Waals interactions were calculated using a $1.0 \mathrm{~nm}$ radius cutoff.

The data obtained from the MD simulations were treated and analyzed with GROMACS and specific codes developed in Python. MDAnalysis (Michaud-Agrawal et al., 2011; Gowers et al., 2016) was employed to preprocess the trajectories, to calculate the water and ion survival probability $[P(\tau)]$ in the SCPN, and the radial distribution functions $[g(\mathrm{r})]$. NumPy (Harris et al., 2020) and Pandas (Mckinney, 2010) were also used to treat and organize data, and Matplotlib (Hunter, 2007) was used for graphical representations. The snapshots and animations from trajectories were made using VMD (Humphrey et al., 1996). $g(\mathrm{r})$ was employed as implemented in MDAnalysis, while $P(\tau)$ was obtained from the following equation:

$$
P(\tau)=\frac{1}{T} \sum_{t=1}^{T} \frac{N(t, t+\tau)}{N(t)},
$$

where $T$ is the maximum time of the simulation, $\tau$ is the time period used as a parameter of the survival probability (a time window after which the probability of a molecule to remain in the cavity is determined), $N(t)$ is the number of molecules inside the $a, \delta$-SCPN at the time $t$, and $N(t, t+\tau)$ is the remaining number of those molecules at $t$ to $\tau$. From this relation, the residence halflife time $\left(\tau_{1 / 2}\right)$ can be estimated as the value of $\tau$ at $P(\tau)=0.5$.

The average velocity of waters and ions over time windows of $10 \mathrm{ps}$, along the whole trajectories (discarding the first $20 \mathrm{~ns}$ ), was determined from the coordinates of the different species between 

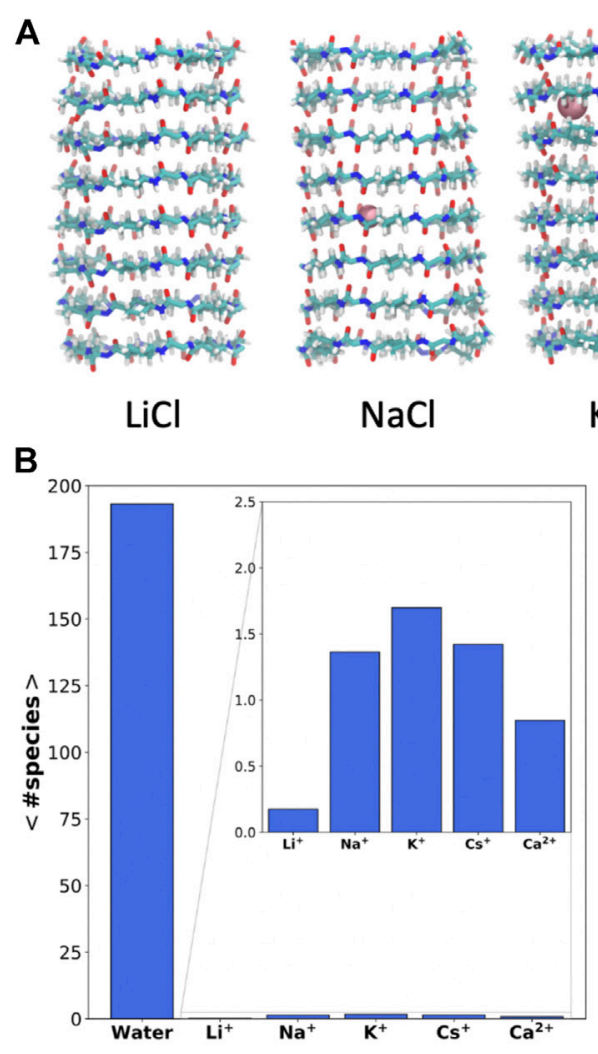
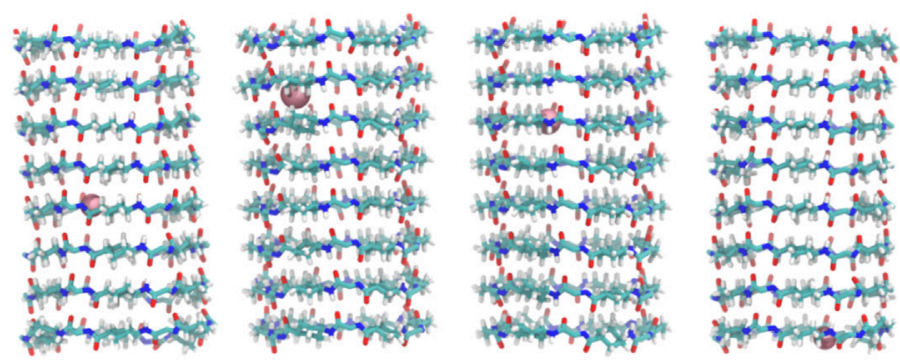

$\mathrm{KCl}$
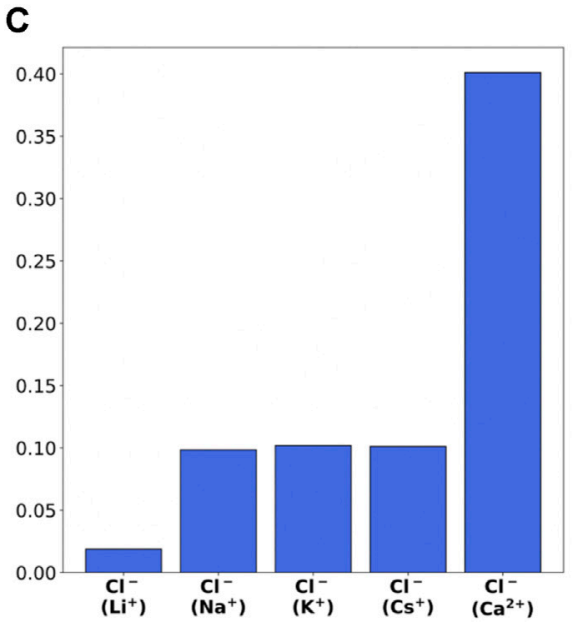

FIGURE 2 | (A) Snapshot of the SCPNs corresponding to the last frame of the MD simulations ( $\mathrm{t}=200 \mathrm{~ns}$ ), including the ions inside (pink) at that time (the lateral chain is not shown for clarity of the image). (B and C) Average number of cations (B) and anions (C) inside the $\alpha, \delta$-SCPN averaged over the last 180 ns of the MD simulations in $\mathrm{LiCl}, \mathrm{NaCl}, \mathrm{KCl}, \mathrm{CsCl}$, and $\mathrm{CaCl}_{2} 0.15 \mathrm{M}$, respectively. Diving into confinement: structure of water and ions inside the $\alpha, \delta$-SCPN.

frames stores in the trajectories. These velocities were associated to each initial position. Given the quasicylindrical geometry of the nanotube, the probability of the positions for water and ions was plotted using cylindrical coordinates $(R, \theta)$, where $R$ is the distance from the axis of the nanotube and $\theta$ is the angle around such axis. The velocities of the same particles were also expressed in cylindrical coordinates and represented as a function of $\mathrm{R}$ or $\theta$.

\section{RESULTS AND DISCUSSION}

\section{Stability of the $\alpha, \delta$-Self-Assembling Cyclic Peptide Nanotubes: The Fortress of the Nanotunnel}

Previous studies with an $\alpha, \delta$-SCPN composed by $c-[L-G l n-$ $\delta$-Ach- $D$-Trp- $\delta$-Ach- $L$-Trp- $\delta$-Ach- $D$-Leu- $\delta$-Ach- $L$-Trp- $\delta$-Ach$D$-Trp- $\delta$-Ach] showed that the nanotube structure is stable in the lipid bilayer environment (Calvelo et al., 2021). The substitution of both Gln and Leu by Trp residues shown in the present work does not have a significant effect on the transmembrane channel stability, as indicated by the low average Root Mean Square Deviations (RMSDs) of the SCPN backbone reached along the trajectories (Supplementary Table 1). The tubular shape is preserved in all the simulations (Figure 2A). The high and constant number of $\mathrm{H}$-bonds between CPs along the channel backbone (Supplementary Table 1) confer rigidity to the whole structure, thus providing a stable scaffold throughout the $\alpha, \delta$-SCPN. The average number of $\mathrm{H}$-bonds between CPs $(78$ in all the simulations) is slightly lower than the maximum that can be theoretically formed ( $12 \mathrm{H}$-bonds by each $\mathrm{CP}$-pair, giving a total of $84 \mathrm{H}$-bonds). This is possibly due to some transient imperfections in the structure and also to the competition with the water molecules inside and outside the channel (Supplementary Table 1), which suggests the dynamic character of the tubular structure.

Despite its significant hydrophobic character, a considerable amount of water ( 195 molecules) can be found inside the $\alpha, \delta$-SCPN (Figure 2B). The amount of water within the nanocylinder volume delimited by the CPs is not significantly altered in the presence of ions (Supplementary Figure 1). On the other hand, whereas almost no $\mathrm{Li}^{+}$cations can penetrate the pore, the $\alpha, \delta$-SCPN admits an average of $1-3 \mathrm{Na}^{+}, \mathrm{K}^{+}$, or $\mathrm{Cs}^{+}$and $1-2$ $\mathrm{Ca}^{2+}$ cations (Figure 2B and Supplementary Figure 2A). The appearance of $\mathrm{Cl}^{-}$anions inside the channels is significant but much lower than that of cations, and no more than a single anion was observed in this volume at the same time (Supplementary 


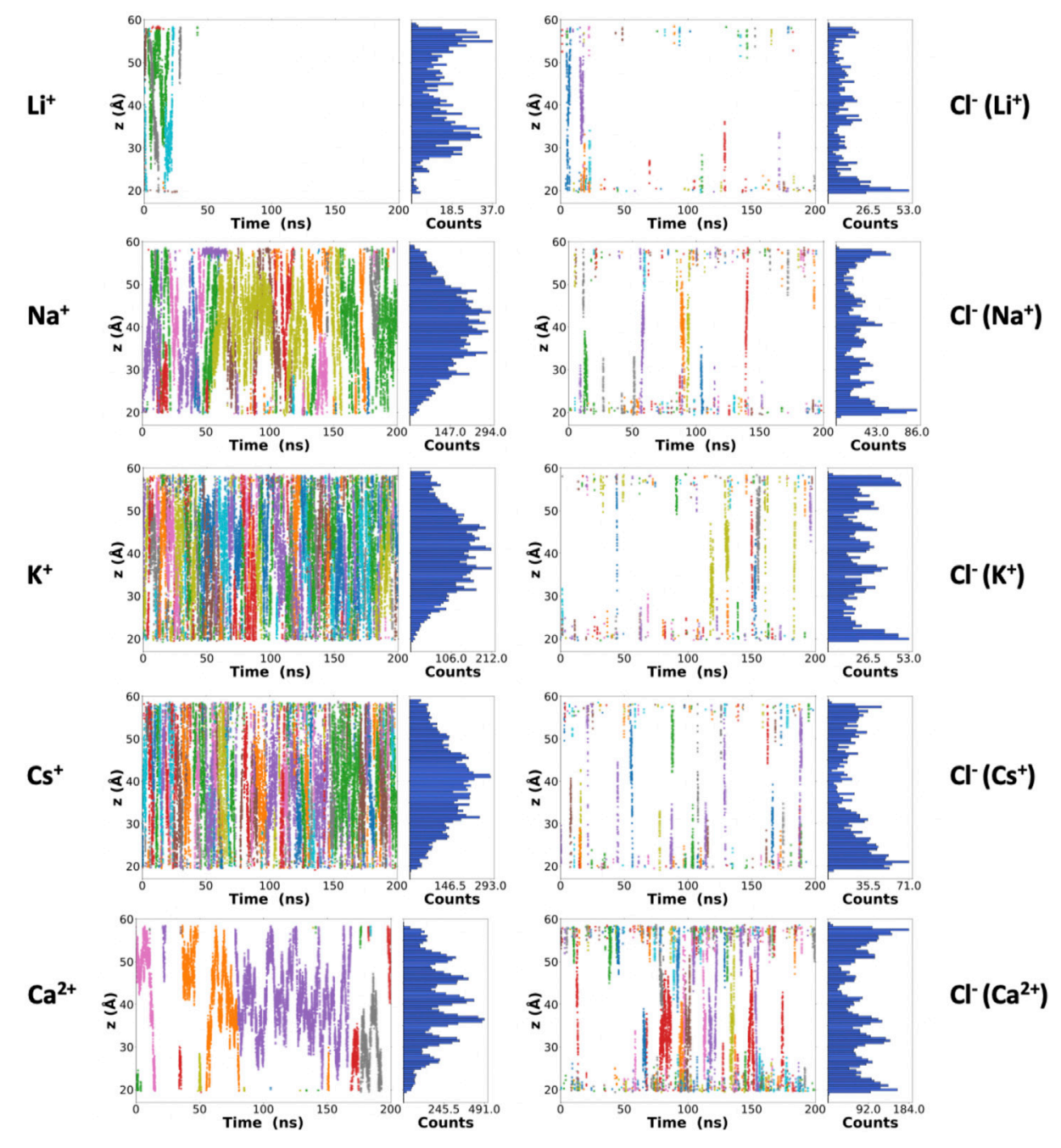

FIGURE 3 |Z-coordinates (along the symmetry axis of the nanotube) for the cations (left) and anions (right) along the 200 ns of the corresponding trajectories. Each color represents a different atom as a function of time. From top to bottom: $\mathrm{LiCl}, \mathrm{NaCl}, \mathrm{KCl}, \mathrm{CsCl}$, and $\mathrm{CaCl}_{2} \mathrm{O} .15 \mathrm{M}$. $\mathrm{A}$ complementary plot, with the number of frames where at least one ion is located in each z-position of the nanotube, is also shown at the right side of each graphic.

Figure 2B). In contrast, no anions at all were observed within the pore of the smaller and less hydrophobic $\alpha, \gamma$-SCPNs (GarcíaFandiño et al., 2012a; Calvelo et al., 2015).

Besides the number of ions inside the channels, it is possible to monitor their positions along the lumen of the corresponding nanotubes for each frame of the trajectory (Figure 3). Some $\mathrm{Li}^{+}$ cations were observed inside the $\alpha, \delta$-SCPN just for the first $25 \mathrm{~ns}$ of simulation. After this time, they appear attached to the lipid phosphates, which prevent their entry into the pore (Supplementary Figure 3). The astounding preference for phosphate groups displayed by $\mathrm{Li}^{+}$ions is in good concordance with previous studies (Kruczek et al., 2017) that predict this behavior. This cation is sequestered by the polar head groups of the lipids in the membrane, thus rendering it unable to traverse the nanotube, at least at the time scale these experiments were carried out. Thus, $\mathrm{Li}^{+}$cations in the nanopore cavity volume will not be considered for the rest of the analysis in this manuscript. As it had been previously observed for this type of channel (Calvelo et al., 2021) and analogously to $\alpha, \boldsymbol{\gamma}$-SCPNs (García-Fandiño et al., 2012a; Garcia-Fandiño et al., 2016), the effective radius is situated in the plane of the $\mathrm{CP}$, whereas the maximum radius is located in the region between the two planes of the rings. However, the transport of $\mathrm{Na}^{+}, \mathrm{K}^{+}$, and $\mathrm{Cs}^{+}$is significantly different to that observed in octameric $\alpha, \gamma$-SCPNs (García-Fandiño et al., 2012a; Calvelo et al., 2015), presumably due not only to the higher hydrophobic character of this nanotube but also to larger radius of these three ions. While the pores formed by $\alpha, \gamma$-SCPNs exhibit regions where the cations spend more time, acting as brakes against the advance of the ions along the pore, the transport of $\mathrm{Na}^{+}, \mathrm{K}^{+}$, and $\mathrm{Cs}^{+}$in $\alpha, \delta$-SCPN takes place in a more continuous way, with a probability distribution centered in the middle of the nanotube (Figure 3). The discrete advance of the cation is still present in the case of $\mathrm{Ca}^{2+}$, where its accumulation in specific zones along 


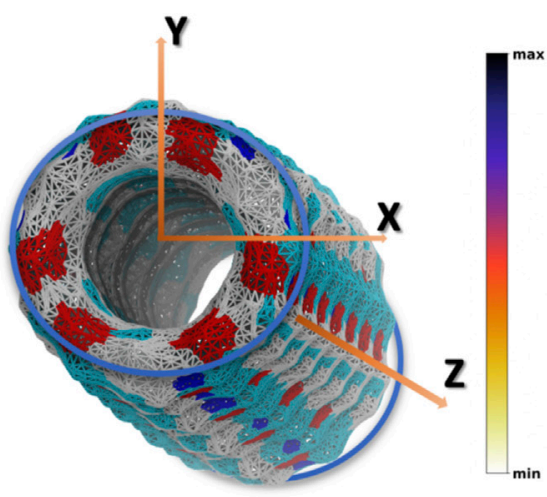

Water

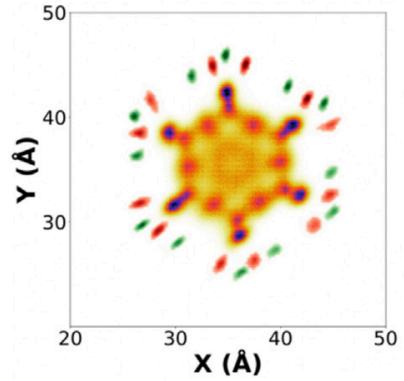

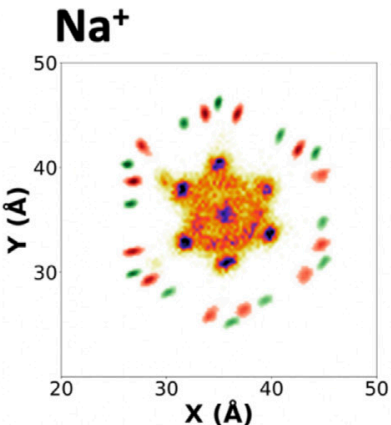

$\mathrm{Ca}^{2+}$

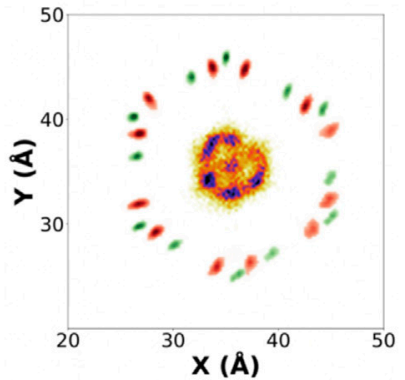

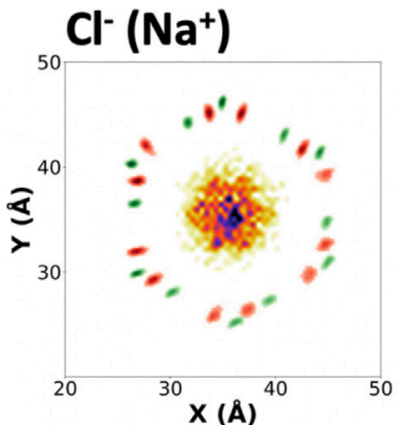

$\mathrm{Cl}^{-}\left(\mathrm{Ca}^{2+}\right)$

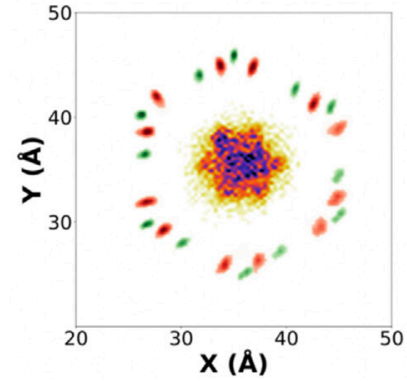

FIGURE 4 | Positional probability distributions represented as heatmaps (increasing from orange to blue) at the $\mathrm{XY}$ plane for $\mathrm{Na}^{+}$, Ca ${ }^{2+}$, and their correspondent counter $\mathrm{Cl}^{-}$anions. Water (from the blank trajectory) is shown at the down left of the figure. The averaged position of alpha carbons (green) and carbonyl groups (red) is also represented as a reference. These 2D-heatmaps were generated using the last 180 ns of the MD simulations.

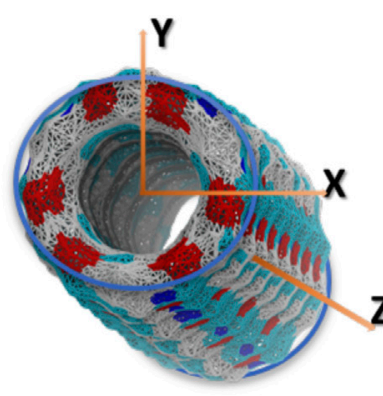

Water

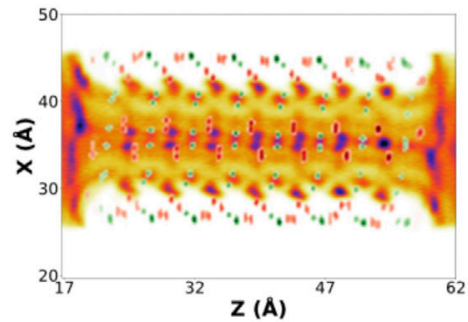

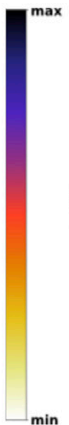
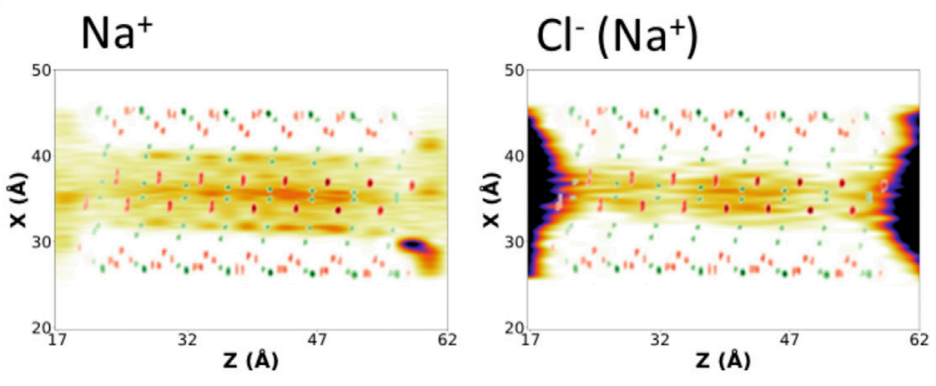

$\mathrm{Ca}^{2+}$

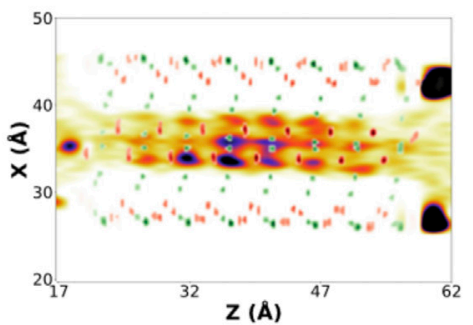

$\mathrm{Cl}^{-}\left(\mathrm{Ca}^{2+}\right)$

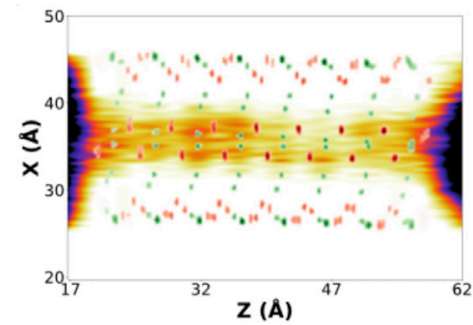

FIGURE 5 | Positional probability distributions represented as heatmaps (increasing from orange to blue) at the $\mathrm{XZ}$ plane for $\mathrm{Na}^{+}$, $\mathrm{Ca}^{2+}$, and their correspondent counter $\mathrm{Cl}^{-}$anions. Water (from the blank trajectory) is shown at the down left of the figure. The averaged position of alpha carbons (green) and carbonyl groups (red) is also represented as a reference. These 2D-heatmaps were generated using the last $180 \mathrm{~ns}$ of the MD simulations. 
the symmetry axis of the channel is much more noticeable than for the rest of the cations. Differences in the distribution functions along the main axis of the nanotube arise from the alternation between the smallest radii, corresponding to the plane of the CPs, and the larger radii regions, corresponding to the interplane sections.

The highest abundance of $\mathrm{Cl}^{-}$inside the channel is observed for the case of the simulation with $\mathrm{CaCl}_{2}$. For this simulation, a single cation remains inside the $\alpha, \delta$-SCPN for long periods of more than 100 ns (Figure 2C and Figure 3). The distributions shown in Figure 3 indicate that a complementary $\mathrm{Cl}^{-}$anion seems to accompany always the $\mathrm{Ca}^{2+}$ cations, suggesting that electrostatic interactions mediate the permeability of the nanotube toward anions. For the rest of the simulations, $\mathrm{Cl}^{-}$ gets only ever so shyly inside the nanotube, also sketching a relatively spatial complementarity along the symmetry axis of the nanotube with respect to the different countercations.

To better understand the distribution of species along the $\alpha, \delta$-SCPN, their probability distribution in two dimensions, obtained from the number of counts at each position with respect to the total amount of counts inside the SCPN, is represented as heatmaps. These $2 \mathrm{D}$ heatmaps have been represented in the transversal XY (Figure 4and Supplementary Figure 4) and longitudinal XZ (Figure 5 and Supplementary Figure 5) or YZ (Supplementary Figure 6) planes. Animations where the view of the heatmap is rotated along the longitudinal plane are available in the supplementary information (Supplementary Animation 1). Despite their larger diameter, compared to $\alpha, \boldsymbol{\gamma}$-SCPNs (García-Fandiño et al., 2012a; Calvelo et al., 2015), the nanoconfined water molecules in the $a, \delta$-SCPN adopt a highly ordered distribution both in the transversal and longitudinal planes. The water spatial distribution over the transversal XY plane reveals a clearly defined six-point star shape, for the blank simulation (Figure 4). It is comprised of concentrical rings where water molecules tend to accumulate. The external ring, forming the tips of the star, corresponds to water directly attached to the $\mathrm{CO}$ and $\mathrm{NH}$ groups of the SCPN backbone via $\mathrm{H}$-bonds (type I, from now on). In particular, most of these water molecules establish $\mathrm{H}$-bonds with the CO groups of Trp (42 $\pm 3 \mathrm{H}$-bonds) and $\delta$-Ach $(18 \pm 3 \mathrm{H}$-bonds) residues and to a lesser extent with the $\mathrm{NH}$ moiety $(5 \pm 1 \mathrm{H}$-bonds with the $\mathrm{NH}$ group of Trp residues and $6 \pm 2 \mathrm{H}$-bonds with the $\mathrm{NH}$ group of $\delta$-Achresidues) (Values Averaged from the Last $50 \mathrm{Ns}$ of the MD Simulations). The following inner ring can be split into two types of water molecules: those bridged to the outer ring (type $I I$ ) and those lying between the former water molecules, in the region next to the two methylene groups of the $\delta$-Ach (type III). Finally, there is an innermost ring, more diffuse than those previously described (type IV). Furthermore, it is worth noticing that, besides the appearance of lobes where water tends to reside, there are regions that water tends to avoid, creating channels with decreased water density. These regions guide the position and diffusion of cations (see below). Water distribution on the $\mathrm{XZ}$ and $\mathrm{YZ}$ planes is also very well defined (Figure 5 and Supplementary Figure 6). The darkest lobes correspond to inter CP plane regions, coexisting with slightly less denser areas belonging to water placed at the CP planes.

The presence of ions in the simulation does not seem to affect the exotic water pattern found in the blank simulation (Supplementary Figure 7). Instead, it seems that this predefined water distribution inside the $\alpha, \delta$-SCPN creates a coordinating scaffold through which the ions move. In this way, the $2 \mathrm{D}$ heatmaps for cations in the $\mathrm{XY}$ plane are complementary to those corresponding to water (Figure 4 and Supplementary Figure 4). Similar to water, ions also exhibit a characteristic 2D distribution heatmap over the SCPN circular plane. $\mathrm{Na}^{+}$forms a reciprocal hexagonal star, with six peaks at the regions in contact with type II and type III waters described before (Figure 4). Overall, the shape of the star becomes more diffuse from $\mathrm{Na}^{+}$to $\mathrm{Cs}^{+}$(Figure 4 and Supplementary Figure 4), a demeanor possibly attributed to the larger ion radius. Thus, bigger cations prefer the central zones of the pore, whereas those smaller are more localized at the borders or inside specific channels where they can fit better. On the other hand, $\mathrm{Ca}^{2+}$ illustrates the influence of charge, rather than radius (as compared with the monovalent cations), on the preferred position of a divalent cation through an $\alpha, \delta$-SCPN (Figure 4). The probability distribution of the divalent cation displays a compact shape in the XY plane, indicating that the ions are confined in the innermost part of the lumen. This suggests that $\mathrm{Ca}^{2+}$ ion movements are restricted to smaller regions than the other cations. The ion disposition along the symmetry axis of the nanopore is also different, depending on its size and charge. While the most populated regions for $\mathrm{Na}^{+}$and $\mathrm{K}^{+}$are those between $\mathrm{CP}$ planes, $\mathrm{Cs}^{+}$and $\mathrm{Ca}^{2+}$ seem to prefer the plane of the CP (Figure 5 and Supplementary Figures 5,6).

The $2 \mathrm{D}$ heatmaps for $\mathrm{Cl}^{-}$suggest a dependence of the anion location and diffusion on their cation counterpart. $\mathrm{Cl}^{-}$ accumulation in the $\mathrm{XY}$ plane reveals a less-defined six-point star, the clearest case being $\mathrm{CaCl}_{2}$ (Figure 4). It is notable how these stars are rotated $60^{\circ}$ with respect to their cation analogues. While positive charges have well-defined channels through which they can diffuse without disrupting the scaffold that water creates inside the SCPN and may even get "lubricated" by coordinating to their oxygens, anions have trouble finding a place to fit and move through, even more so if they are as bulky as a chloride. On top of that steric hindrance, the only possible coordination that a $\mathrm{Cl}^{-}$ can have with water is through its hydrogens. This further disrupts the water scaffold, as it would imply an inversion of the conformation of some water molecules. This flip would render the oxygen atom from the coordinated water molecule close to the oxygen atoms of the waters forming the scaffold, creating an electrostatic repulsion, and destabilizing a very stable lattice by entailing an energetic penalty. On the other hand, the anions' 2D heatmaps of the averaged positions along the longitudinal planes show very similar profiles for all the simulations, the only difference being the intensity of the population (Figure 5 and Supplementary Figures 5,6).

In order to gather information about the surroundings of each cation within the $a, \delta$-SCPN, Radial Distribution Functions (RDFs) were calculated (Figure 6 and Supplementary Figure 8). They display a clear first water coordination sphere 

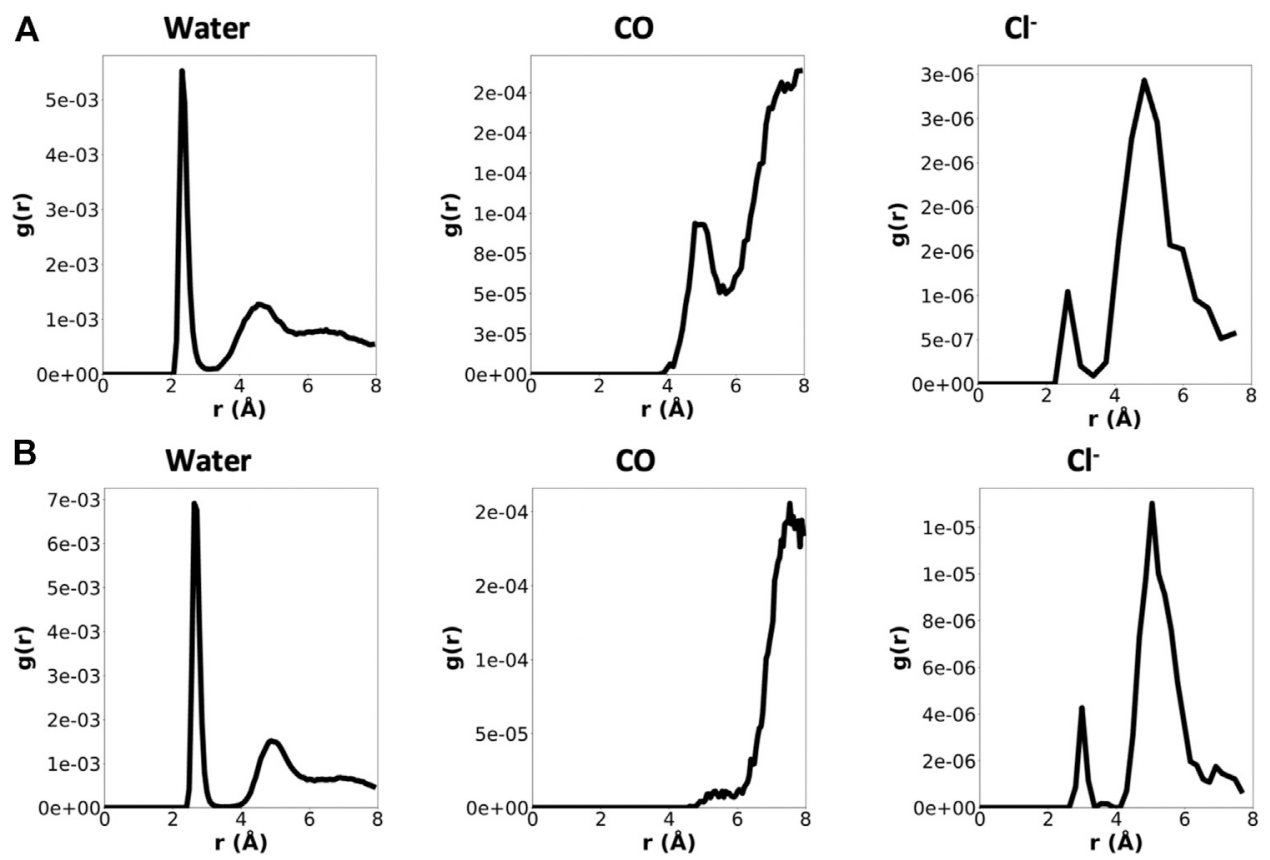

FIGURE 6 |RDFs for $\mathrm{Na}^{+} \mathbf{( A )}$ and $\mathrm{Ca}^{2+}$ (B) considering different contributions (water, $\mathrm{CO}$ groups, and $\mathrm{Cl}^{-}$ions), normalized by the number of cations and number of contributions. Just the last 180 ns were considered for the analysis. Note that the scales for the three contributions are not the same.

A

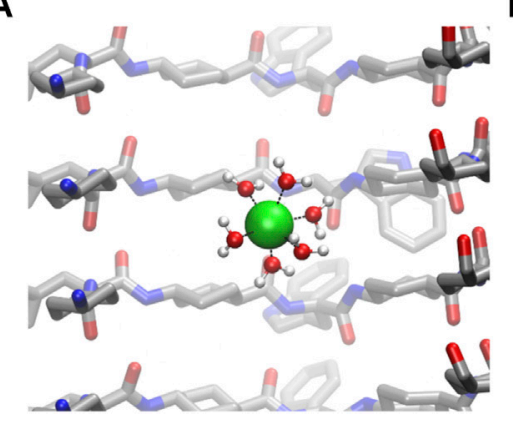

B

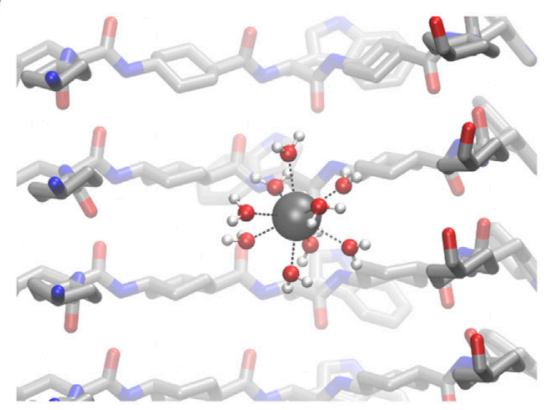

FIGURE 7 | Details of the first sphere of coordination of confined $\mathrm{Na}^{+}(\mathbf{A})$ and $\mathrm{Ca}^{2+} \mathbf{( B )}$.

TABLE 1 | Average number (and standard deviation) of contacts between water, oxygens from $\mathrm{CO}$, and $\mathrm{Cl}^{-}$anions participating in the first coordination sphere of each cation. The radius of the first coordination sphere was taken from the RDF calculations. Just the last $180 \mathrm{~ns}$ were considered for the analysis.

\begin{tabular}{lccc} 
Cation & Water & Co & $\mathbf{C l}^{-}$ \\
\hline $\mathrm{Na}^{+}$ & $5.5 \pm 0.56$ & $0.00 \pm 0.03$ & $0.00 \pm 0.04$ \\
$\mathrm{~K}^{+}$ & $6.21 \pm 0.75$ & $0.01 \pm 0.13$ & $0.01 \pm 0.08$ \\
$\mathrm{Cs}^{+}$ & $5.26 \pm 0.94$ & $0.00 \pm 0.08$ & $0.01 \pm 0.11$ \\
$\mathrm{Ca}^{2+}$ & $8.83 \pm 0.4$ & $0.00 \pm 0.01$ & $0.01 \pm 0.10$
\end{tabular}

for all species, which migrates toward bigger distances for ions presenting larger atomic radii. None of the cations studied tend to directly coordinate to the $\mathrm{CO}$ groups of the amino acid backbone in this first coordination sphere at distances $\sim 4-6 \AA$. Except for
$\mathrm{Ca}^{2+}$, these $\mathrm{CO}$ groups do participate in their second coordination sphere. On the other hand, some $\mathrm{Cl}^{-}$ions do directly contribute to the first coordination sphere of all the cations, but this contribution is poor.

The calculation of the number of contacts between cations and their possible contributions to the first coordination sphere (calculated from the RDF values) also confirms that CO groups do not coordinate directly to cations (Figure 7). The number of water molecules in the first coordination sphere of all cations is very similar to the theoretical maximum in bulk, suggesting that the energetic penalty that these ions pay to enter into the tube is low (Table 1 and Figure 7) (Ohtomo and Arakawa, 1979; Bakó et al., 2002; Varma and Rempe, 2006). Almost no $\mathrm{Cl}^{-}$ion or oxygen from $\mathrm{CO}$ was found at the first coordination sphere distance of any cation, confirming that this situation is unlikely. 
TABLE 2 |Residence half-life time $\left(\tau_{1 / 2}\right)$ (ns) calculated from the survival probability $[P(\tau)]$ evolution for cations and anions in each simulation (Supplementary Figure 9).

\begin{tabular}{lcc}
$\tau_{\mathbf{1 / 2}}(\mathbf{n s})$ & Cation & Anion \\
\hline $\mathrm{NaCl}$ & 3.1 & 0.2 \\
$\mathrm{KCl}$ & 1.2 & 0.2 \\
$\mathrm{CsCl}$ & 1.0 & 0.2 \\
$\mathrm{CaCl}_{2}$ & 4.6 & 0.3
\end{tabular}

It was observed that the number of coordinating water molecules inside the nanotube often exceeds the theoretical maximum (6) for the cations. This situation is clear for $\mathrm{K}^{+}$, where the average number is $6.21 \pm 0.75$. One plausible explanation is that nanoconfined systems behave differently than their bulk relatives. SCPNs can be approximated to a hollow cylinder, where one dimension (vertical, Z) is much larger than the other two (circular plane, XY). This leaves a space where two degrees of freedom become restricted, and thus, movement in those directions becomes hampered. It is easy to imagine, then, that in such a system with particles moving inside, collisions are more likely to occur than in a free unrestricted medium. This might lead to a compression of the first sphere of coordination for cations, forcing the ligands toward the metallic center and affecting the ligand exchange process. It should also be noted by the higher SDs on the inner number of waters (Table 1), when compared with those of the bulk analysis, that these "hypercoordinated" states might only happen for very short periods of time, being unstable.

\section{Movement Into Confinement: Dynamics of Water and Ions Inside the $a, \delta$-Self-Assembling Cyclic Peptide Nanotubes}

For the proper characterization of the $a, \delta-S C P N$ as a transmembrane channel, it is interesting to study how the different molecules move through the nanotube, in addition to characterize their most likely location. From the survival probability (Supplementary Figure 9) and the corresponding half-life time values (Table 2), it can be appreciated that the most short-lived species in the $\alpha, \delta$-SCPN is water, followed by $\mathrm{Cs}^{+}, \mathrm{K}^{+}$, $\mathrm{Na}^{+}$, and $\mathrm{Ca}^{2+}$. The residence half-time for $\mathrm{Cl}^{-}$anions is significantly lower, even for the simulation in $\mathrm{CaCl}_{2}$.

The distributions of average velocities between saved frames ( $10 \mathrm{ps}$ far from each other) of the different ions and water molecules were determined from the MD trajectories. The results for the solvent and ions show that water molecules reach the highest average speeds over this windows time, although there is a significant contribution indicating the existence of slower water molecules in the corresponding distribution, presumably those involved in type $I \mathrm{H}$-bonds (Figure 8). Just as the presence of ions did not seem to affect the overall water pattern inside the pore, they do not seem to alter their velocity distribution either. For a given valence, cations with bigger radii tend to diffuse faster along

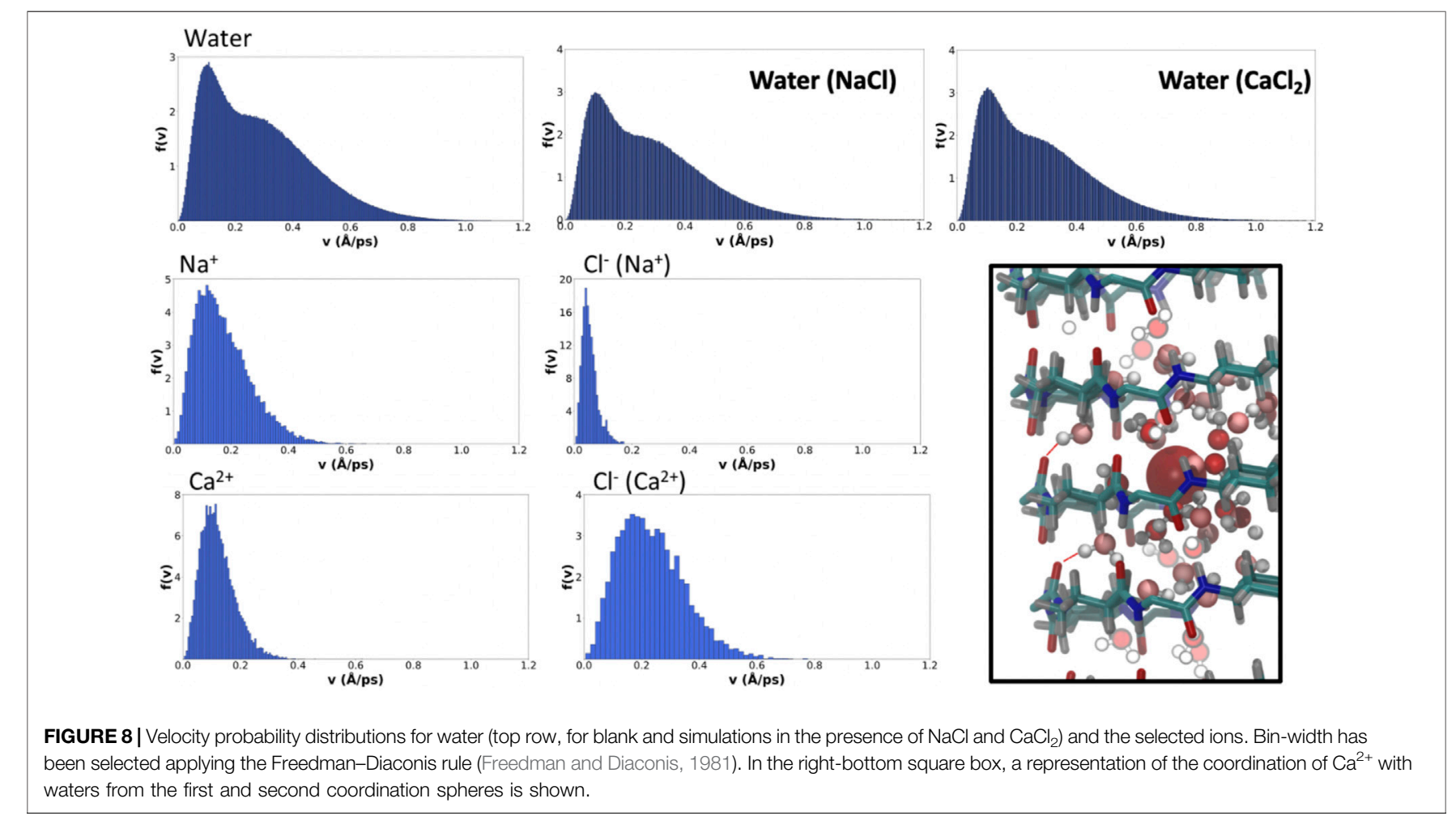


the $\alpha, \delta$-SCPN, as it can be observed from the broadening toward higher speeds of the distributions from $\mathrm{Na}^{+}$to $\mathrm{Cs}^{+}$(Figure 8 and Supplementary Figure 10). Cation speed seems to be directly related to its degree of accommodation in the nanotube cavity. $\mathrm{Na}^{+}$, for example, seems to be relatively comfortable inside the pore, compared to the other ions, as can be inferred from the ordered 2D heatmap pattern corresponding to the XY plane (Figure 4). The spatial disposition into a full set of lobes in each of the points of the star and an extra central lobe allows this ion to find several places where its complete coordination sphere can be maintained without disturbing the general order of confined water. The alternation among these positions in the XY plane seems to slow down its movement along the nanotube symmetry axis, justifying its longer residence times. On the other hand, whereas $\mathrm{K}^{+}$and $\mathrm{Cs}^{+}$display roughly the same amount of coordinating waters than $\mathrm{Na}^{+}$(Table 1), their bigger radii demand a global larger space inside the pore. This hinders the ability to reach a stable scaffold of $\mathrm{H}$-bonds with the confined water in the channel and forces it to move around less favorable locations, thus promoting the diffusion through the nanotube. This is also in agreement with the more diffuse star-like shapes these two ions present in the XY planes compared to $\mathrm{Na}^{+}$ (Figure 4). The comparison of the results for $\mathrm{Ca}^{2+}$ and $\mathrm{Na}^{+}$ (Figure 8) provides evidence of the important role of the charge over the radius or mass of the cation. Both ions have a very similar radii, but the charge of the $\mathrm{Ca}^{2+}$ forces its coordination sphere to accommodate a larger number of water molecules (Figure 7), thus extending it to longer distances. Despite being restricted to a more central position in the nanotube, thus suggesting a lower interaction with the nanotube walls, $\mathrm{Ca}^{2+}$ exhibits the higher residence times of all the studied cations (Table 2 and Supplementary Figure 9). This apparent contradiction is, nonetheless, supported by the fact that $\mathrm{Ca}^{2+}$, together with its set of coordinate water molecules, fits better at the plane of the $\mathrm{CPs}$ than between $\mathrm{CP}$ planes. In that arrangement, the second coordination sphere is composed just by waters, as confirmed by the RDF and coordination numbers. Many of these water molecules are of type $I$, thus coordinated to $\mathrm{CO}$ groups from the SCPN backbone (Figure 8, square).

The velocity distribution for $\mathrm{Cl}^{-}$is similar for all the monovalent cation simulations, much lower than those corresponding to the cationic counterparts (Figure 8). This fact, together with their short residence times in the nanotube, explains the fact that they hardly explore any position in the XY plane in most of the cases (Figure 4 and Supplementary Figure 4), crossing the channel in a very short time. It is again in the simulation with $\mathrm{Ca}^{2+}$ where a remarkably different demeanor is observed in the movement of the $\mathrm{Cl}^{-}$anion (Figure 8). In this case, not only the distribution is broader and with a slower decay rate than in the rest of the simulations but it also reaches higher speeds than the cation compared to the rest of cases. Their higher speed is also accompanied by a slightly longer residence time (Table 2 and Supplementary Figure 9). The explanation for this difference relies on the influence that the counterion has in the movement of $\mathrm{Cl}^{-}$. Although it does not occupy the $\mathrm{Ca}^{2+}$ first coordination sphere, its movement is coupled to the correspondent cation, having an influence in the diffusion along the nanotube.
The existence of more than one possible velocity distributions for one single species suggests that there should be more than one associated behaviors. We propose that those different conducts should be related to the position of the particle inside the nanotube. The velocity of particles located in the inner part of the nanotube is expected to be different from that located in one of the lobes. To demonstrate this hypothesis and taking advantage of the tubular shape of the nanopore, 2D heatmap distributions of positions and velocities in cylindrical coordinates $(R, \theta)$ were created (Figure 9 and Supplementary Figure 11). The velocity distributions for both the water molecules and cations display a clear correlation with the distance to center of the pore (R). There is also some dependence on $\theta$, although to a lesser extent, generally corresponding to the slower motion at the lobes near the walls of the SCPN. Water, which displays a much better sampling than ions, gives the most detailed picture. It is possible to appreciate that the outermost lobes, from 6-8 $\AA$, correspond to the slowest molecules (type I). Out of this area, the region from 5-6 ̊̊ exhibits slightly higher velocities with a broader span. This corresponds to the type II waters of the middle ring, defined in the previous section. In the region from 3-6 $\AA$ and intercalated between the lobes in the $\theta$-axis, the type III waters of the middle ring are observed. These water molecules reach higher velocities, and the shape of the distribution is narrower than that of the type IV waters. Finally, corresponding to the innermost region of the SCPN, a change in slope in the $\mathrm{R}$ correlation diagram, from $3 \AA$ and down, is seen. This indicates that the water molecules falling inside this region will move faster than the previous ones, and the dependence on $\mathrm{R}$ is more pronounced than for the previously described waters.

Again, $\mathrm{Na}^{+}$(Figure 9), $\mathrm{K}^{+}$, and $\mathrm{Cs}^{+}$(Supplementary Figure 10) seem to present similarities in their behavior. For $\mathrm{K}^{+}$and $\mathrm{Cs}^{+}$, the dependence of the velocities on $\mathrm{R}$ is clear, displaying a confined slow regime in the lobes and a tendency for velocity to increase as we get closer to the center of the SCPN. $\mathrm{Na}^{+}$displays the same behavior, although the dependence with $\mathrm{R}$ is weaker. Also, its innermost region seems to have a drop on the velocities, possibly due to its central high-density region (Figure 4). In line with other analysis, $\mathrm{Ca}^{2+}$ (Figure 9) also displays a completely different behavior. The correlation between the speeds and R is not as clear as for the other cations, but a sort of discontinuity in the velocity as a function of $\mathrm{R}$ can be observed around $1.75 \AA$ (Figure 9). Also, the relationship between $\theta$ and the velocity distribution seems to be slightly smoother than for the rest of the cations.

The velocity distributions of anions are similar for all the simulations. $\mathrm{Cl}^{-}$movement does not clearly depend on its position inside the nanotube (Figure 9 and Supplementary Figure 11). Only the case for the simulation with $\mathrm{CaCl}_{2}$ seems to have a small drift toward slightly higher speeds for the smallest values of $\mathrm{R}$. This behavior is in agreement with what was previously proposed about the movement of the anions: their diffusion along the nanotube is not free, but it is driven by the cations present in the system. Thus, the transport of $\mathrm{Cl}^{-}$by cations with the same charge will be very similar to each other, while changing the valence of the cation a high impact on the velocities reached by the anion is expected, compared to that caused by the positional distribution within the SCPN. 

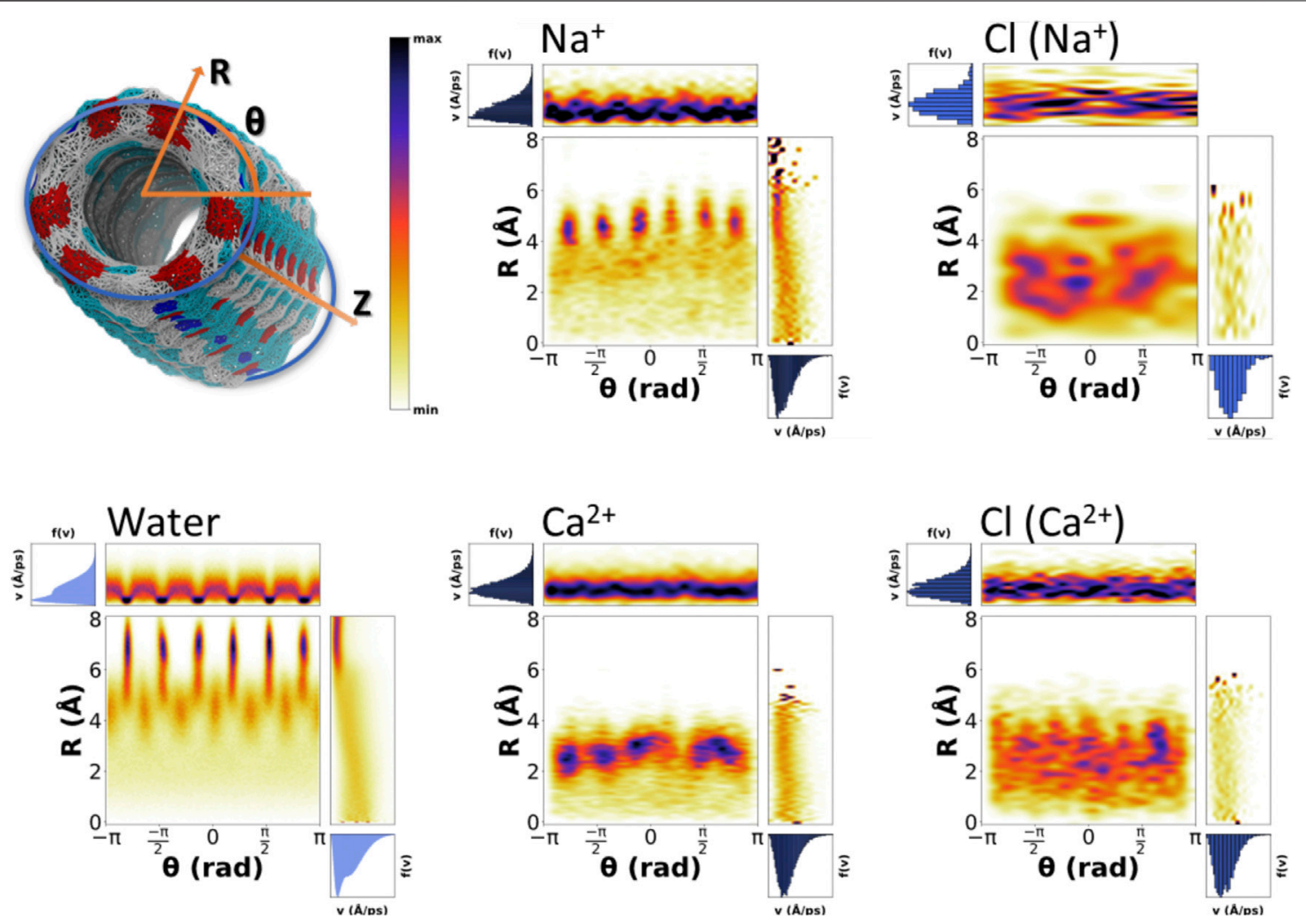

FIGURE 9|Position-velocity correlation diagrams for water (bottom left), selected cations (middle top, $\mathrm{Na}^{+}$, and down, $\mathrm{Ca}^{2+}$ ), and correspondent counterions (right column). The central plot of each diagram corresponds to the probability of positions as a function of cylindrical coordinates (R, $\theta)$, the top plot is the velocity distribution as a function of the angle, and the right plot is the velocity distribution as a function of the distance to the center of the nanotube symmetry axis. The analysis was generated using the last $180 \mathrm{~ns}$ of the MD simulations.

\section{CONCLUSION}

The observations of an underwater expedition through the nanoconfined space of a transmembrane $\alpha, \delta$-SCPN by wearing a diving equipment in the form of MD simulations are described here. The presence of $\delta$-aminocycloalkanecarboxylic acids in the CP sequence confers a partially hydrophobic character to the pore, which has a critical impact on the behavior of the nanoconfined ions and water molecules. MD simulations of an $\alpha, \delta$-SCPN composed of CPs with the sequence $c$ - $[L-(\operatorname{Trp}-\delta$-Ach $)-(D-\operatorname{Trp}-\delta-A c h)-L-(\operatorname{Trp}-$ $\delta$-Ach)-D-(Trp- $\delta$-Ach)- $L-(\operatorname{Trp}-\delta-A c h)-D-(\operatorname{Trp}-\delta-A c h)]$ in the presence of different salt compositions (blank, $\mathrm{LiCl}, \mathrm{NaCl}, \mathrm{KCl}$, $\mathrm{CsCl}$, and $\mathrm{CaCl}_{2}$ ) indicate that they are stable when inserted into a lipid bilayer. The hybrid hydrophilic-hydrophobic environment created by the nature of the residues in the sequence defines the disposition of water molecules inside the channel. Nanoconfined water in the $\alpha, \delta$-SCPN adopts a highly ordered distribution both in the transversal and longitudinal planes. The water spatial distribution over the transversal plane reveals a clearly defined six-point star shape, formed by at least four water types (type I-IV), with a different diffusion behavior. While $\mathrm{Li}^{+}$is sequestered by the lipid phosphate groups which prevent its internalization in the nanopore, $\mathrm{Na}^{+}, \mathrm{K}^{+}, \mathrm{Cs}^{+}$, and $\mathrm{Ca}^{2+}$ were observed to go inside the channel at different rates, proportions, and locations. Globally, no more than three ions of the same class appear within the channel at the same time in any of the simulations, the average number being between one and three in most cases. The appearance of $\mathrm{Cl}^{-}$anions inside the channels is scarce compared to that of cations although, unlike happened with the smaller and less-hydrophobic $a, \gamma$-SCPNs, a small proportion was observed.

Ions accommodate inside the cylinder trying to disturb as little as possible the exotic pattern that water molecules create inside the channel. The ions with less impact in the water organization remain for a longer time inside the nanotube, as revealed by the survival probability profile and the corresponding residence half-life times. This is achieved in the first place by $\mathrm{Ca}^{2+}$ and then by $\mathrm{Na}^{+}, \mathrm{K}^{+}$, and $\mathrm{Cs}^{+}$, in this order. In all cases, the first water coordination spheres for all the cations are completely preserved, being the type of molecules that belong to its second sphere of coordination that will define the advance of the cations along the symmetry axis of the nanotube.

\section{DATA AVAILABILITY STATEMENT}

The datasets generated and analyzed for this study are included in the article/Supplementary Material. All initial coordinates, topologies and mdp files needed for reproducing these simulations are available in Zenodo through the following link: https://zenodo.org/record/ 
4732313. Further inquiries can be directed to the corresponding author.

\section{AUTHOR CONTRIBUTIONS}

AB-G performed the MD simulations and analyzed the data. AP, MC, and PG discussed the data. RG-F conceived the study and discussed the data. All authors discussed the results and contributed to the writing of the paper.

\section{FUNDING}

This work was supported by the Spanish Agencia Estatal de Investigación (AEI) and the ERDF (PID2019-111126RB-100, RTI2018-098795-A-I00, and PID2019-111327GB-I00) and by

\section{REFERENCES}

Abraham, M. J., Murtola, T., Schulz, R., Páll, S., Smith, J. C., and Hess, B. (2019). GROMACS: High Performance Molecular Simulations Through Multi-Level Parallelism From Laptops to Supercomputers,. SoftwareX 1-2 (19), 19-25. doi:10.5281/ZENODO.3243834

Amorín, M., Castedo, L., and Granja, J. R. (2003). New Cyclic Peptide Assemblies with Hydrophobic Cavities: The Structural and Thermodynamic Basis of a New Class of Peptide Nanotubes. J. Am. Chem. Soc. 125 (10), 2844-2845. doi:10.1021/ja0296273

Aryal, P., Sansom, M. S. P., and Tucker, S. J. (2015). Hydrophobic Gating in Ion Channels. J. Mol. Biol. 427, 121-130. doi:10.1016/j.jmb.2014.07.030

Bakó, I., Hutter, J., and Pálinkás, G. (2002). Car-Parrinello Molecular Dynamics Simulation of the Hydrated Calcium Ion. J. Chem. Phys. 117 (21), 9838-9843. doi:10.1063/1.1517039

Bogdanowicz, K. A., Bhosale, S. V., Li, Y., Vankelecom, I. F. J., Garcia-Valls, R., Reina, J. A., et al. (2016). Mimicking Nature: Biomimetic Ionic Channels. J. Membr. Sci. 509, 10-18. doi:10.1016/j.memsci.2016.02.038

Bong, D. T., Clark, T. D., Granja, J. R., and Ghadiri, M. R. (2001). Self-Assembling Organic Nanotubes. Angew. Chem. Int. Ed. 40 (6), 988-1011. doi:10.1002/15213773(20010316)40:6<988::aid-anie9880>3.0.co;2-n

Brea, R. J., Reiriz, C., and Granja, J. R. (2010). Towards Functional Bionanomaterials Based on Self-Assembling Cyclic Peptidenanotubes. Chem. Soc. Rev. 39, 1448-1456. doi:10.1039/b805753m

Bussi, G., Donadio, D., and Parrinello, M. (2007). Canonical Sampling through Velocity Rescaling. J. Chem. Phys. 126 (1), 014101. doi:10.1063/1.2408420

Calvelo, M., Granja, J. R., and Garcia-Fandino, R. (2019). Competitive DoubleSwitched Self-Assembled Cyclic Peptide Nanotubes: A Dual Internal and External Control. Phys. Chem. Chem. Phys. 21 (37), 20750-20756. doi:10.1039/c9cp02327e

Calvelo, M., Lamas, A., Guerra, A., Amorín, M., Garcia-Fandino, R., and Granja, J. R. (2020). Parallel Versus Antiparallel $\beta$-Sheet Structure in Cyclic Peptide Hybrids Containing $\gamma$ - or $\delta$-Cyclic Amino Acids. Chem. Eur. J. 26 (26), 5846-5858. doi:10.1002/chem.201905554

Calvelo, M., Lynch, C. I., Granja, J. R., Sansom, M. S. P., and Garcia-Fandiño, R. (2021). Effect of Water Models on Transmembrane Self-Assembled Cyclic Peptide Nanotubes. ACS Nano 15, 7053-7064. doi:10.1021/acsnano.1c00155

Calvelo, M., Vázquez, S., and García-Fandiño, R. (2015). Molecular Dynamics Simulations for Designing Biomimetic Pores Based on Internally Functionalized Self-Assembling a, $\gamma$-peptide Nanotubes. Phys. Chem. Chem. Phys. 17 (43), 28586-28601. doi:10.1039/c5cp04200c

Chen, J., Li, Q., Wu, P., Liu, J., Wang, D., Yuan, X., et al. (2020). Cyclic $\gamma$-Peptides with Transmembrane Water Channel Properties. Front. Chem. 8, 368. doi:10.3389/fchem.2020.00368 the Xunta de Galicia and the ERDF (ED431F 2020/05, ED431C 2017/25, and Centro singular de investigación de Galicia accreditation 2016-2019, ED431G/09). MC thanks Xunta de Galicia for a predoctoral fellowship (ED481A-2017/ 068). RG-F thanks Ministerio de Ciencia, Innovación y Universidades, for a "Ramón y Cajal" contract (RYC-2016-20335). PFG thanks the Spanish Ministry of Economy and Competitiveness and the European Social Fund for his predoctoral research grant, reference BES-2016-076761. All calculations were carried out at the Centro de Supercomputación de Galicia.

\section{SUPPLEMENTARY MATERIAL}

The Supplementary Material for this article can be found online at: https://www.frontiersin.org/articles/10.3389/fchem.2021.704160/ full\#supplementary-material

Clark, T. D., Buehler, L. K., and Ghadiri, M. R. (1998). Self-Assembling Cyclic $\beta 3$ Peptide Nanotubes as Artificial Transmembrane Ion Channels. J. Am. Chem. Soc. 120 (4), 651-656. doi:10.1021/ja972786f

Clark, T. D., Buriak, J. M., Kobayashi, K., Isler, M. P., McRee, D. E., and Ghadiri, M. R. (1998). Cylindrical $\beta$-Sheet Peptide Assemblies. J. Am. Chem. Soc. 120 (35), 8949-8962. doi:10.1021/ja981485i

Claro, B., Bastos, M., and Garcia-Fandino, R. (2018). "Design and Applications of Cyclic Peptides," in Peptide Applications in Biomedicine, Biotechnology and Bioengineering in Editor Koutsopoulos S. (Sawston, United Kingdom: Woodhead Publishing), 87-129. doi:10.1016/B978-0-08-100736-5.00004-1

De Santis, P., Morosetti, S., and Rizzo, R. (1974). Conformational Analysis of Regular Enantiomeric Sequences. Macromolecules Vol. 7, 52-58. doi:10.1021/ ma60037a011

Essmann, U., Perera, L., Berkowitz, M. L., Darden, T., Lee, H., and Pedersen, L. G. (1995). A Smooth Particle Mesh Ewald Method. J. Chem. Phys. 103 (19), 8577-8593. doi:10.1063/1.470117

Freedman, D., and Diaconis, P. (1981). On the Histogram as a Density Estimator:L 2 Theory. Z. Wahrscheinlichkeitstheorie Verw Gebiete 57 (4), 453-476. doi:10.1007/BF01025868

García-Fandiño, R., Amorín, M., Castedo, L., and Granja, J. R. (2012a). Transmembrane Ion Transport by Self-Assembling $\alpha, \gamma$-peptide Nanotubes. Chem. Sci. 3 (11), 3280-3285. doi:10.1039/c2sc21068a

García-Fandiño, R., Amorín, M., and Granja, J. R. (2012b). "Synthesis of Supramolecular Nanotubes," in Supramolecular Chemistry Editors Steed J. W., Gale P. A., Atwood J. L. (Chichester, UK: John Wiley \& Sons). doi:10.1002/9780470661345.smc081

Garcia-Fandiño, R., Calvelo, M., and Granja, J. R. (2017). "Pore- and ChannelForming Peptides and Their Mimetics," in Comprehensive Supramolecular Chemistry IIEditors SteedJ. W., GaleP. A., AtwoodJ. L. (Elsevier Inc.), 4, 539-573. doi:10.1016/B978-0-12-409547-2.12546-6

García-Fandiño, R., Granja, J. R., Orozco, M., and Orozco, M. (2009). Theoretical Characterization of the Dynamical Behavior and Transport Properties of $\alpha, \gamma$-Peptide Nanotubes in Solution. J. Am. Chem. Soc. 131 (43), 15678-15686. doi:10.1021/ja903400n

Garcia-Fandiño, R., Piñeiro, Á., Trick, J. L., and Sansom, M. S. P. (2016). Lipid Bilayer Membrane Perturbation by Embedded Nanopores: A Simulation Study. ACS Nano 10 (3), 3693-3701. doi:10.1021/acsnano.6b00202

Ghadiri, M. R., Granja, J. R., and Buehler, L. K. (1994). Artificial Transmembrane Ion Channels from Self-Assembling Peptide Nanotubes. Nature 369 (6478), 301-304. doi:10.1038/369301a0

Ghadiri, M. R., Granja, J. R., Milligan, R. A., McRee, D. E., and Khazanovich, N. (1993). Self-Assembling Organic Nanotubes Based on a Cyclic Peptide Architecture. Nature 366 (6453), 324-327. doi:10.1038/366324a0 
Gowers, R. J., Linke, M., Barnoud, J., E Reddy, T. J., Melo, M. N., Seyler, S. L., Dománski, J., Dotson, D. L., Buchoux, S., Kenney, I. M., and Beckstein, O. (2016). "MDAnalysis: A Python Package for the Rapid Analysis of Molecular Dynamics Simulations," in Proceedings of the 15th Python in Science Conference, Austin, TX. doi:10.25080/majora-629e541a-00e

Granja, J. R., and Reza Ghadiri, M. (1994). Channel-Mediated Transport of Glucose across Lipid Bilayers. J. Am. Chem. Soc. 116, 10785-10786. doi:10.1021/ja00102a054

Harris, C. R., Millman, K. J., van der Walt, S. J., Gommers, R., Virtanen, P., Cournapeau, D., et al. (2020). Array Programming with NumPy. Nature 585, 357-362. doi:10.1038/s41586-020-2649-2

Hess, B., Bekker, H., Berendsen, H. J. C., and Fraaije, J. G. E. M. (1997). LINCS: A Linear Constraint Solver for Molecular Simulations. J. Comput. Chem. 18 (12), 1463-1472. doi:10.1002/(sici)1096-987x(199709)18:12<1463::aid-jcc4>3.0.co;2-h

Humphrey, W., Dalke, A., and Schulten, K. (1996). VMD: Visual Molecular Dynamics. J. Mol. Graphics 14 (1), 33-38. doi:10.1016/0263-7855(96)00018-5

Hunter, J. D. (2007). Matplotlib: A 2D Graphics Environment. Comput. Sci. Eng. 9 (3), 90-95. doi:10.1109/MCSE.2007.55

Jämbeck, J. P. M., and Lyubartsev, A. P. (2012a). An Extension and Further Validation of an All-Atomistic Force Field for Biological Membranes. J. Chem. Theor. Comput. 8 (8), 2938-2948. doi:10.1021/ct300342n

Jämbeck, J. P. M., and Lyubartsev, A. P. (2012b). Derivation and Systematic Validation of a Refined All-Atom Force Field for Phosphatidylcholine Lipids. J. Phys. Chem. B 116 (10), 3164-3179. doi:10.1021/jp212503e

Joung, I. S., and Cheatham, T. E. (2008). Determination of Alkali and Halide Monovalent Ion Parameters for Use in Explicitly Solvated Biomolecular Simulations. J. Phys. Chem. B 112 (30), 9020-9041. doi:10.1021/jp8001614

Klauda, J. B., Venable, R. M., Freites, J. A., O'Connor, J. W., Tobias, D. J., Mondragon-Ramirez, C., et al. (2010). Update of the CHARMM All-Atom Additive Force Field for Lipids: Validation on Six Lipid Types. J. Phys. Chem. B 114 (23), 7830-7843. doi:10.1021/jp101759q

Kruczek, J., Chiu, S.-W., Jakobsson, E., and Pandit, S. A. (2017). Effects of Lithium and Other Monovalent Ions on Palmitoyl Oleoyl Phosphatidylcholine Bilayer. Langmuir 33 (4), 1105-1115. doi:10.1021/acs.langmuir.6b04166

Lamas, A., Guerra, A., Amorín, M., and Granja, J. R. (2018). New Self-Assembling Peptide Nanotubes of Large Diameter Using $\delta$-amino Acids. Chem. Sci. 9 (43), 8228-8233. doi:10.1039/c8sc02276c

Lindorff-Larsen, K., Piana, S., Palmo, K., Maragakis, P., Klepeis, J. L., Dror, R. O., et al. (2010). Improved Side-Chain Torsion Potentials for the Amber Ff99SB Protein Force Field. Proteins 78 (8), 1950-1958. doi:10.1002/prot.22711

Lynch, C. I., Rao, S., and Sansom, M. S. P. (2020). Water in Nanopores and Biological Channels: A Molecular Simulation Perspective. Chem. Rev.American Chem. Soc. September 120, 10298-10335. doi:10.1021/acs.chemrev.9b00830

Mckinney, W. (2010). "Data Structures for Statistical Computing in Python," in Proceedings of the 9th Python in Science Conference. 51-56. doi:10.25080/majora92bf1922-00a

Garcia-Fandino, R., Amorin, M., Castedo, L., and Granja, J. R. (2012). Transmembrane Ion Transport by Self-Assembling $\alpha, \gamma$-Peptide Nanotubes. Chem. Sci. 3 (4), 3280-3285. doi:10.1039/C2SC21068A

Michaud-Agrawal, N., Denning, E. J., Woolf, T. B., and Beckstein, O. (2011). MDAnalysis: A Toolkit for the Analysis of Molecular Dynamics Simulations. J. Comput. Chem. 32 (10), 2319-2327. doi:10.1002/jcc.21787

Montenegro, J., Ghadiri, M. R., and Granja, J. R. (2013). Ion Channel Models Based on Self-Assembling Cyclic Peptide Nanotubes. Acc. Chem. Res. 46 (12), 2955-2965. doi:10.1021/ar400061d

Ohtomo, N., and Arakawa, K. (1979). Neutron Diffraction Study of Aqueous Ionic Solutions. I. Aqueous Solutions of Lithium Chloride and Caesium Chloride. Bcsj 52 (10), 2755-2759. doi:10.1246/bcsj.52.2755

Parrinello, M., and Rahman, A. (1981). Polymorphic Transitions in Single Crystals: A New Molecular Dynamics Method. J. Appl. Phys. 52 (12), 7182-7190. doi:10.1063/1.328693
Price, D. J., and Brooks, C. L. (2004). A Modified TIP3P Water Potential for Simulation with Ewald Summation. J. Chem. Phys. 121 (20), 10096-10103. doi:10.1063/1.1808117

Rao, S., Lynch, C. I., Klesse, G., Oakley, G. E., Stansfeld, P. J., Tucker, S. J., et al. (2018). Water and Hydrophobic Gates in Ion Channels and Nanopores. Faraday Discuss. 209 (0), 231-247. doi:10.1039/c8fd00013a

Reiriz, C., Amorín, M., García-Fandiño, R., Castedo, L., and Granja, J. R. (2009). a, $\gamma$-Cyclic Peptide Ensembles with a Hydroxylated Cavity. Org. Biomol. Chem. 7 (21), 4358-4361. doi:10.1039/b911247m

Rodríguez-Vázquez, N., Amorín, M., and Granja, J. R. (2017). Recent Advances in Controlling the Internal and External Properties of Self-Assembling Cyclic Peptide Nanotubes and Dimers. Org. Biomol. Chem. 15, 4490-4505. doi:10.1039/c7ob00351j

Rodríguez-Vázquez, N., García-Fandiño, R., Amorín, M., and Granja, J. R. (2016). Self-assembling $\alpha, \gamma$-cyclic Peptides that Generate Cavities with Tunable Properties. Chem. Sci. 7 (1), 183-187. doi:10.1039/c5sc03187g

Rodriguez-Vazquez, N., Lionel Ozores, H., Guerra, A., Gonzalez-Freire, E., Fuertes, A., Panciera, M., et al. (2014). Membrane-Targeted Self-Assembling Cyclic Peptide Nanotubes. Curr. Top. Med. Chem. 14, 2647-2661. doi:10.2174/ 1568026614666141215143431

Sánchez-Quesada, J., Sun Kim, H., and Ghadiri, M. R. (2001). A Synthetic PoreMediated Transmembrane Transport of Glutamic Acid. Angew. Chem. Int. Ed. 40 (13), 2503-2506. doi:10.1002/1521-3773(20010702)40:13<2503::AIDANIE2503>3.0.CO;2-E

Seebach, D., Matthews, J. L., Meden, A., Wessels, T., Baerlocher, C., and McCusker, L. B. (1997). Cyclo-?-peptides: Structure and Tubular Stacking of Cyclic Tetramers of 3-aminobutanoic Acid as Determined from Powder Diffraction Data. Helv. Chim. Acta 80 (1), 173-182. doi:10.1002/hlca.19970800116

Tabata, Y., Mitani, S., Uji, H., Imai, T., and Kimura, S. (2019). The Effect of Macrodipole Orientation on the Piezoelectric Response of Cyclic $\beta$-peptide Nanotube Bundles on Gold Substrates. Polym. J. 51 (6), 601-609. doi:10.1038/ s41428-019-0169-4

Tagliazucchi, M., and Szleifer, I. (2015). Transport Mechanisms in Nanopores and Nanochannels: Can We Mimic Nature?. Mater. Today 18, 131-142. doi:10.1016/j.mattod.2014.10.020

Varma, S., and Rempe, S. B. (2006). Coordination Numbers of Alkali Metal Ions in Aqueous Solutions. Biophysical Chem. 124 (3), 192-199. doi:10.1016/ j.bpc.2006.07.002

Yazdani, M., Jia, Z., and Chen, J. (2020). Hydrophobic Dewetting in Gating and Regulation of Transmembrane Protein Ion Channels. J. Chem. Phys. 153, 110901. doi:10.1063/5.0017537

Zheng, S. P., Huang, L. B., Sun, Z., and Barboiu, M. (2021). Self-Assembled Artificial Ion-Channels toward Natural Selection of Functions. Angew. Chem. Int. Ed. 60 (2), 566-597. doi:10.1002/anie.201915287

Conflict of Interest: The authors declare that the research was conducted in the absence of any commercial or financial relationships that could be construed as a potential conflict of interest.

Publisher's Note: All claims expressed in this article are solely those of the authors and do not necessarily represent those of their affiliated organizations, or those of the publisher, the editors and the reviewers. Any product that may be evaluated in this article, or claim that may be made by its manufacturer, is not guaranteed or endorsed by the publisher.

Copyright (c) 2021 Blanco-González, Calvelo, Garrido, Amorín, Granja, Piñeiro and Garcia-Fandino. This is an open-access article distributed under the terms of the Creative Commons Attribution License (CC BY). The use, distribution or reproduction in other forums is permitted, provided the original author(s) and the copyright owner(s) are credited and that the original publication in this journal is cited, in accordance with accepted academic practice. No use, distribution or reproduction is permitted which does not comply with these terms. 